K. Ihara and H. Ochiai

Nagoya Math. J.

Vol. 189 (2008), 49-62

\title{
SYMMETRY ON LINEAR RELATIONS FOR MULTIPLE ZETA VALUES
}

\author{
KENTARO IHARA AND HIROYUKI OCHIAI*
}

\begin{abstract}
We find a symmetry for the reflection groups in the double shuffle space of depth three. The space was introduced by Ihara, Kaneko and Zagier and consists of polynomials in three variables satisfying certain identities which are connected with the double shuffle relations for multiple zeta values. Goncharov has defined a space essentially equivalent to the double shuffle space and has calculated the dimension. In this paper we relate the structure among multiple zeta values of depth three with the invariant theory for the reflection groups and discuss the dimension of the double shuffle space in this view point.
\end{abstract}

\section{$\S 1$. Introduction}

The multiple zeta value (MZV for short) is a number defined via convergent series

$$
\zeta\left(k_{1}, k_{2}, \ldots, k_{n}\right)=\sum_{m_{1}>m_{2}>\cdots>m_{n}>0} \frac{1}{m_{1}^{k_{1}} m_{2}^{k_{2}} \cdots m_{n}^{k_{n}}} \in \mathbf{R},
$$

where $\left(k_{1}, k_{2}, \ldots, k_{n}\right)$ is an index set of positive integers with $k_{1}>1$. Here $n$ is called the depth and $k=k_{1}+\cdots+k_{n}$ the weight of the MZV. Let $\mathcal{Z}=\bigoplus_{k \geqslant 0} \mathcal{Z}_{k}$ be the graded vector space over $\mathbf{Q}$, where $\mathcal{Z}_{k}$ is the $\mathbf{Q}$-vector space generated by MZV's of weight $k$. It is known that $\mathcal{Z}$ is equipped with a filtered graded algebra structure over $\mathbf{Q}: \mathcal{Z}_{k}^{(n)} \mathcal{Z}_{k^{\prime}}^{\left(n^{\prime}\right)} \subset \mathcal{Z}_{k+k^{\prime}}^{\left(n+n^{\prime}\right)}$, where $\mathcal{Z}_{k}^{(n)}$ is the subspace spanned by MZV's of weight $k$ and depth $\leqslant n$. The algebra $\mathcal{Z}$ is deeply connected with the theory of mixed motives and Galois representation on the fundamental group of a curve (see [3], [4], [5], [10] for further details). There is a conjectural formula due to Broadhurst and Kreimer in [1], giving the numbers $D_{k, n}$ of algebra generators of $\mathcal{Z}$ of weight $k$ and depth $n$. (The precise statement is in Section 4.)

\section{Received July 10, 2006.}

Revised February 9, 2007.

2000 Mathematics Subject Classification: Primary 11M41; Secondary 11M06, $40 B 05$.

* The research of the second author is supported in part by a Grant-in-Aid for Scientific Research (B) 15340005 from Japan Society for the Promotion of Science. 
In [8], Kaneko, Zagier and the first author define a Q-vector space $D S h_{n}(d)$ (called double shuffle space) for each $n, d$ whose dimension gives an upper bound of the numbers $D_{d+n, n}$. The space $D S h_{n}(d)$ consists of homogeneous polynomials of degree $d$ in $n$ variables with rational coefficients which satisfy certain identities. These identities are connected with two kinds of product structures in $\mathcal{Z}$, which is called double shuffle products. For example, for the case $n=2$, it is a necessary and sufficient condition for $f \in \mathbf{Q}\left[x_{1}, x_{2}\right]$ of homogeneous degree $d$ to belong to $D S h_{2}(d)$ to satisfy the following conditions:

$$
f\left(x_{1}, x_{2}\right)+f\left(x_{2}, x_{1}\right)=0, \quad f\left(x_{1}+x_{2}, x_{1}\right)+f\left(x_{1}+x_{2}, x_{2}\right)=0 .
$$

The general definition of $D S h_{n}(d)$ is given in the next section. In an unpublished manuscript 'Multiple zeta values (1995)' (see also [9]), Zagier shows that the dimension of $D S h_{2}(d)$ is 0 for odd $d$ and $\left[\frac{d}{6}\right]$ for even $d$ and that $D_{d+2,2}$ equals 0 for odd $d$ and is less than or equals $\left[\frac{d}{6}\right]$ for even $d$. A further development is given in [8]. In [7], the dimension of a space $S h C_{n}(d)$ is determined for small $n \leqslant 5$. The space is introduced in [8] and contains $D S h_{n}(d)$ as a subspace. So this gives an upper bound of $D_{d+n, n}$, but is not the best estimate. In [2], as a result in case of $n=3$, Goncharov defines a space, called dihedral Lie coalgebra, essentially equivalent to the double shuffle space and proves that the number $D_{d+3,3}$ of generators of depth 3 is 0 for odd $d$ and is less than or equals $\left[\frac{d^{2}-1}{48}\right]$ for even positive $d$.

In this paper, we concentrate our attention on the double shuffle space $D S h_{3}$ of depth 3 . We find a symmetry of the reflection group of type $B_{3}$ on the double shuffle space $D S h_{3}$ and as a corollary we have an expression of the space $D S h_{3}$ in terms of the invariants under the reflection groups.

Here is the brief organization of the paper. We begin with the review of the definition of $D S h_{n}$ according to [8] in Section 2.1, and in Section 2.2 we show that $D S h_{3}$ can be regarded as the intersection of the kernels of two averaging maps associated to the reflection group and its subgroups. The key result in this section is Theorem 7. In Section 3 we show that the images of the averaging maps above have trivial intersection. In the last section, we summarize our main result with a short exact sequence and determine the dimension of $D S h_{3}(d)$. This leads to the estimate of $D_{d+3,3}$ which is compatible with Broadhurst-Kreimer conjecture. 


\section{$\S 2$. Double shuffle space of depth three}

\subsection{Preliminary}

In this subsection, we review the definition of the space $D S h_{n}$ according to $[8]$.

Let $\mathfrak{S}_{n}$ be the symmetric group of degree $n$ and $\mathbf{Z}\left[\mathfrak{S}_{n}\right]$ its group ring. We denote by $\mathbf{Q}[\boldsymbol{x}]$ the polynomial algebra $\mathbf{Q}\left[x_{1}, \ldots, x_{n}\right]$ in $n$ variables with rational coefficients and by $\mathbf{Q}[\boldsymbol{x}]_{(d)}$ its subspace of homogeneous polynomials of degree $d$. We define a right action of $\mathfrak{S}_{n}$ on these spaces by permutation of variables: $(f \mid \sigma)\left(x_{1}, \ldots, x_{n}\right)=f\left(x_{\sigma^{-1}(1)}, \ldots, x_{\sigma^{-1}(n)}\right)$. This action extends to an action of $\mathbf{Z}\left[\mathfrak{S}_{n}\right]$ additively. For each integer $l$ with $1 \leqslant l<n$, define the $l^{\text {th }}$ shuffle element by $s h_{l}=\sum \sigma \in \mathbf{Z}\left[\mathfrak{S}_{n}\right]$, where the sum runs over the element $\sigma \in \mathfrak{S}_{n}$ satisfying $\sigma(1)<\cdots<\sigma(l)$ and $\sigma(l+1)<\cdots<\sigma(n)$. Then the double shuffle space $D S h_{n}$ is defined by

$$
D S h_{n}=\left\{f \in \mathbf{Q}[\boldsymbol{x}]|f| s h_{l}=f^{\sharp} \mid s h_{l}=0 \text { for } 1 \leqslant l<n\right\}
$$

where $f^{\sharp}$ is given by $f^{\sharp}\left(x_{1}, \ldots, x_{n}\right)=f\left(x_{1}+x_{2}+\cdots+x_{n}, x_{2}+\cdots+\right.$ $\left.x_{n}, \ldots, x_{n-1}+x_{n}, x_{n}\right)$. We write $D S h_{n}(d)$ for its homogeneous part of degree $d$.

Let $C=(1,2, \ldots, n) \in \mathfrak{S}_{n}$ be a cyclic element of order $n$. Using the identity $s h_{l} C^{l}=s h_{n-l}$ for any $l$, we can remove the half of defining relations of $D S h_{n}$. For example, $D S h_{3} \subset \mathbf{Q}\left[x_{1}, x_{2}, x_{3}\right]$ is defined by the relations

$$
\left\{\begin{array}{l}
\left(f \mid s h_{1}\right)\left(x_{1}, x_{2}, x_{3}\right)=f\left(x_{1}, x_{2}, x_{3}\right)+f\left(x_{2}, x_{1}, x_{3}\right)+f\left(x_{2}, x_{3}, x_{1}\right)=0, \\
\left(f^{\sharp} \mid s h_{1}\right)\left(x_{1}, x_{2}, x_{3}\right)=f^{\sharp}\left(x_{1}, x_{2}, x_{3}\right)+f^{\sharp}\left(x_{2}, x_{1}, x_{3}\right)+f^{\sharp}\left(x_{2}, x_{3}, x_{1}\right)=0 .
\end{array}\right.
$$

In [8], it is proved that $\operatorname{dim} D S h_{n}(d)$ gives an upper bound of the number $D_{d+n, n}$ of algebra generators of $\mathcal{Z}$ of weight $d+n$ and depth $n$ and that $D S h_{n}(d)=\{0\}$ for odd $d$. In this paper we discuss the structure of $D S h_{3}(d)$ for even $d$.

\subsection{Reflection group symmetry}

In this subsection, we prove that $D S h_{3}(d)$ with even $d$ is embedded in the algebra of invariant polynomials for the reflection group of type $B_{3}$ and the embedded image is the intersection of the kernels of two averaging maps.

For later use, we first extend the action of $\mathfrak{S}_{3}$ to an action of $G L_{3}(\mathbf{Q})$. For $S \in G L_{3}(\mathbf{Q})$ and $f \in \mathbf{Q}[\boldsymbol{x}]$ with $\boldsymbol{x}=\left(x_{1}, x_{2}, x_{3}\right)$, we define $(f \mid S)(\boldsymbol{x}):=$ $f\left(\boldsymbol{x} S^{-1}\right)$, which agrees with the above action of $\mathfrak{S}_{3}$ if the element $\sigma$ of $\mathfrak{S}_{3}$ 
is identified with permutation matrix (we also denote it by $\sigma$ for simplicity) in $G L_{3}(\mathbf{Q})$ characterized by $\boldsymbol{x} \sigma^{-1}=\left(x_{\sigma^{-1}(1)}, x_{\sigma^{-1}(2)}, x_{\sigma^{-1}(3)}\right)$. This action also extends to that of group $\operatorname{ring} \mathbf{Z}\left[G L_{3}(\mathbf{Q})\right]$. If we put $P \in G L_{3}(\mathbf{Q})$ by

$$
P=\left[\begin{array}{ccc}
1 & 0 & 0 \\
-1 & 1 & 0 \\
0 & -1 & 1
\end{array}\right], \quad P^{-1}=\left[\begin{array}{lll}
1 & 0 & 0 \\
1 & 1 & 0 \\
1 & 1 & 1
\end{array}\right]
$$

then $f^{\sharp}=f \mid P$ for $f \in \mathbf{Q}[\boldsymbol{x}]$.

Let $W$ be the subgroup of $G L_{3}(\mathbf{Q})$ generated by the permutation matrices and diagonal matrices whose entries are \pm 1 . Note that $W \cong \mathfrak{S}_{3} \ltimes\{ \pm 1\}^{3}$, which is of order $3 ! \times 2^{3}=48$, and is isomorphic to the reflection group of type $B_{3}$. Let $W^{\prime}$ be the subgroup of $W$ (of order 16) which consists of the matrices whose $(2,2)$-entry is \pm 1 .

Define the matrix $R:=J-2 I$, where $J$ is the matrix of size 3 whose entries are all 1 , and $I$ is the unit matrix. Then $R \in G L_{3}(\mathbf{Q})$ and $R^{-1}=$ $(J-I) / 2$.

TheOrem 1. For any $f \in D S h_{n}(d)$ with even $d$, put $g:=f \mid R$. Then $g \in \mathbf{Q}[\boldsymbol{x}]^{W^{\prime}}$ where $\mathbf{Q}[\boldsymbol{x}]^{W^{\prime}}$ is the invariant subalgebra for $W^{\prime}$.

The theorem will be proved later.

Lemma 2. The group $W^{\prime}$ is generated by $-I, T$ and $Q^{-1} T Q: W^{\prime}=$ $\left\langle-I, T, Q^{-1} T Q\right\rangle$, where

$$
T=\left[\begin{array}{lll}
0 & 0 & 1 \\
0 & 1 & 0 \\
1 & 0 & 0
\end{array}\right], \quad Q=\left[\begin{array}{ccc}
1 & 0 & 1 \\
0 & 1 & 0 \\
-1 & 0 & 1
\end{array}\right] .
$$

Proof. It is clear since $Q^{-1} T Q=\operatorname{diag}(-1,1,1)$.

Proposition 3. For any $f \in \mathbf{Q}[\boldsymbol{x}]$ and $g=f \mid R$, we have

(i) $f\left|s h_{1}=0 \Longleftrightarrow g\right| s h_{1}=0$,

(ii) $f^{\sharp}\left|s h_{1}=0 \Longleftrightarrow g\right| Q^{-1} s h_{1}=0$.

Proof. Use the equations $J^{2}=3 J$ and $\sigma J=J \sigma=J$ for any permutation matrix $\sigma$, it is easy to verify that $R^{ \pm 1} \sigma R^{\mp 1}=\sigma$. In particular we have $R^{ \pm 1} \operatorname{sh}_{1} R^{\mp 1}=s h_{1}$. For (i), we have

$$
f\left|s h_{1}=0 \Longleftrightarrow g\right| R^{-1} s h_{1} R=g \mid s h_{1}=0 .
$$


For (ii), using an interesting identity $2 R^{-1} P=Q^{-1} R$,

$$
f^{\sharp}\left|s h_{1}=0 \Longleftrightarrow g\right| R^{-1} \operatorname{Psh}_{1}=0 \Longleftrightarrow g\left|Q^{-1} R s h_{1} R^{-1}=g\right| Q^{-1} s_{1}=0 .
$$

This proves the proposition.

Proof of Theorem 1. By Lemma 2, it is enough to show the invariance of $g$ for $-I, T$ and $Q^{-1} T Q$. First, $g \mid(-I)=(-1)^{d} g=g$ since $d$ is even. Next, it is easy to check the equation $\operatorname{sh}_{1}(I-T)=I-T$. From this and Proposition 3, we have

$$
g|(I-T)=g| s h_{1}(I-T)=\left(g \mid s h_{1}\right) \mid(I-T)=0 .
$$

Similarly we have

$$
\begin{aligned}
g \mid\left(I-Q^{-1} T Q\right) & =g\left|Q^{-1}(I-T) Q=g\right| Q^{-1} \operatorname{sh}_{1}(I-T) Q \\
& =\left(g \mid Q^{-1} s_{1}\right) \mid(I-T) Q=0 .
\end{aligned}
$$

Let us define the groups by $\bar{W}=Q^{-1} W Q$ and $\overline{W^{\prime}}=Q^{-1} W^{\prime} Q$.

Proposition 4. We have $W^{\prime}=\overline{W^{\prime}}$.

Proof. By Lemma 2, we have $\overline{W^{\prime}}=\left\langle-I, Q^{-1} T Q, Q^{-2} T Q^{2}\right\rangle$. It is easy to see $W^{\prime}=\overline{W^{\prime}}$, because $Q^{-2} T Q^{2}=\left[\begin{array}{ccc}0 & 0 & -1 \\ 0 & 1 & 0 \\ -1 & 0 & 0\end{array}\right]$.

COROLlary 5. The action of $Q$ gives a linear automorphism on $\mathbf{Q}[\boldsymbol{x}]^{W^{\prime}}=\mathbf{Q}[\boldsymbol{x}]^{\overline{W^{\prime}}}$.

Corollary 6. We have $W \cap \bar{W}=W^{\prime}$.

In the next section we will prove that the intersection of two invariant polynomial algebras $\mathbf{Q}[\boldsymbol{x}]^{W}$ and $\mathbf{Q}[\boldsymbol{x}]^{\bar{W}}$ is trivial.

Let $\pi: \mathbf{Q}[\boldsymbol{x}]^{W^{\prime}} \rightarrow \mathbf{Q}[\boldsymbol{x}]^{W}$, and $\bar{\pi}: \mathbf{Q}[\boldsymbol{x}]^{W^{\prime}} \rightarrow \mathbf{Q}[\boldsymbol{x}]^{\bar{W}}$ be the averaging maps defined by

$$
\pi(g):=\sum_{\sigma \in W^{\prime} \backslash W} g\left|\sigma, \quad \bar{\pi}(g):=\sum_{\sigma \in \overline{W^{\prime}} \backslash \bar{W}} g\right| \sigma=\sum_{\sigma \in W^{\prime} \backslash W} g \mid Q^{-1} \sigma Q,
$$


where $\sigma$ runs over the set of left-coset representatives of $W^{\prime} \backslash W$ or $\overline{W^{\prime}} \backslash \bar{W}$.

Since one can choose the set of terms of $s h_{1}$ as a set of coset representatives of $W^{\prime} \backslash W$, we obtain that

$$
\pi(g)=g\left|s h_{1}, \quad \bar{\pi}(g)=g\right| Q^{-1} \operatorname{sh}_{1} Q .
$$

We denote the part of degree $d$ of $\pi$ and $\bar{\pi}$ by $\pi_{(d)}$ and $\bar{\pi}_{(d)}$ respectively.

It is well-known (e.g., Chap. 3 of [6]) that the ring of invariants under the reflection group is isomorphic to the polynomial ring and that the degrees of generators are read off from the exponents. To be more explicit, we have

$$
\begin{aligned}
\mathbf{Q}[\boldsymbol{x}]^{W} & =\mathbf{Q}\left[x_{1}^{2}+x_{2}^{2}+x_{3}^{2}, x_{1}^{2} x_{2}^{2}+x_{2}^{2} x_{3}^{2}+x_{3}^{2} x_{1}^{2}, x_{1}^{2} x_{2}^{2} x_{3}^{2}\right], \\
\mathbf{Q}[\boldsymbol{x}]^{W^{\prime}} & =\mathbf{Q}\left[x_{1}^{2}+x_{3}^{2}, x_{2}^{2}, x_{1}^{2} x_{3}^{2}\right] .
\end{aligned}
$$

We have the decomposition of $\mathbf{Q}[\boldsymbol{x}]^{W^{\prime}}$ as a $\mathbf{Q}[\boldsymbol{x}]^{W_{-}}$or $\mathbf{Q}[\boldsymbol{x}]^{\bar{W}}$-module

$$
\begin{aligned}
\mathbf{Q}[\boldsymbol{x}]^{W^{\prime}} & =\mathbf{Q}[\boldsymbol{x}]^{W} \oplus g_{2} \mathbf{Q}[\boldsymbol{x}]^{W} \oplus g_{4} \mathbf{Q}[\boldsymbol{x}]^{W} \\
& =\mathbf{Q}[\boldsymbol{x}]^{\bar{W}} \oplus \overline{g_{2}} \mathbf{Q}[\boldsymbol{x}]^{\bar{W}} \oplus \overline{g_{4}} \mathbf{Q}[\boldsymbol{x}]^{\bar{W}},
\end{aligned}
$$

where $g_{2}=x_{1}^{2}+x_{3}^{2}-2 x_{2}^{2}, g_{4}=x_{1}^{2} x_{2}^{2}+x_{2}^{2} x_{3}^{2}-2 x_{1}^{2} x_{3}^{2}$ and $\overline{g_{2}}=g_{2} \mid Q$ and $\overline{g_{4}}=g_{4} \mid Q$. Then $\frac{1}{3} \pi$ and $\frac{1}{3} \bar{\pi}$ are the projections to the first components of (4) and (5) respectively.

TheOREM 7. The action of $R(f \mapsto g=f \mid R)$ gives a linear isomorphism from the space $D S h_{3}(d)$ with even $d$ to $\operatorname{ker} \pi_{(d)} \cap \operatorname{ker} \bar{\pi}_{(d)}$.

Proof. This follows directly from Theorem 1, Proposition 3 and equation (1).

\section{$\S 3 . \quad$ Triviality of the kernel}

In this section, we prove that a polynomial which is $W$-invariant and $\bar{W}$-invariant should be constant. Slightly more generally, we prove that a continuous $W$ and $\bar{W}$ invariant function on $\mathbf{R}^{3}$ should be constant. In Section 3.1, we give a statement of the invariants for a general subgroup of index finite. In Section 3.2, we define a group $\Gamma$ for a general size $n$ and prove to be of finite index. We go back to the setting with $W$ and $\bar{W}$ in Section 3.3. 


\subsection{Invariants}

Proposition 8. Let $\Gamma \subset G L_{n}(\mathbf{Z})$ be a group with finite index. Let $f$ be a continuous function on $\mathbf{R}^{n}$ and suppose $f$ is $\Gamma$-invariant. Then $f$ is constant.

Proof. Let $G L_{n}(\mathbf{Z})=\bigcup_{j=1}^{N} g_{j} \Gamma$ be a coset decomposition. Let us take and fix an $\boldsymbol{x} \in \mathbf{R}^{n}$. We will prove $f(\boldsymbol{x})=f(\mathbf{0})$. It is enough to prove that for any $\varepsilon>0$ we have $|f(\boldsymbol{x})-f(\mathbf{0})|<\varepsilon$. Take an $\varepsilon>0$. Then, since $f$ is continuous,

(i) there exists a $\delta>0$ such that

$$
\begin{aligned}
& \left|f\left(\boldsymbol{z} g_{j}\right)-f(\mathbf{0})\right|<\varepsilon / 2 \\
& \qquad \text { for all } j=1, \ldots, N \text { and for all } \boldsymbol{z} \in \mathbf{R}^{n} \text { with }\|\boldsymbol{z}\|<\delta,
\end{aligned}
$$

where $\|\boldsymbol{z}\|=\max \left\{\left|z_{1}\right|, \ldots,\left|z_{n}\right|\right\}$ for $\boldsymbol{z}=\left(z_{1}, \ldots, z_{n}\right) \in \mathbf{R}^{n}$,

(ii) there exists $\boldsymbol{y}=\left(y_{1}, \ldots, y_{n}\right) \in \mathbf{R}^{n}$ such that $|f(\boldsymbol{x})-f(\boldsymbol{y})|<\varepsilon / 2$ and that $y_{1}, \ldots, y_{n}$ are linearly independent over $\mathbf{Q}$.

The following Lemma 9 implies that there exists a $g \in G L_{n}(\mathbf{Z})$ such that $\|\boldsymbol{y} g\|<\delta$. In particular we can apply (i) for $\boldsymbol{z}=\boldsymbol{y} g$. We can write $g^{-1}=g_{j} \gamma$ with some $\gamma \in \Gamma$ and $j(1 \leqslant j \leqslant N)$. Then

$$
f(\boldsymbol{y})=f\left(\boldsymbol{z} g^{-1}\right)=f\left(\boldsymbol{z} g_{j} \gamma\right)=f\left(\boldsymbol{z} g_{j}\right) .
$$

Hence

$$
|f(\boldsymbol{x})-f(\mathbf{0})| \leqslant|f(\boldsymbol{x})-f(\boldsymbol{y})|+\left|f\left(\boldsymbol{z} g_{j}\right)-f(\mathbf{0})\right|<\varepsilon,
$$

which proves the desired assertion.

Lemma 9. Let $n \geqslant 2$ be an integer. Let $\boldsymbol{y}=\left(y_{1}, \ldots, y_{n}\right) \in \mathbf{R}^{n}$ such that $y_{1}, \ldots, y_{n}$ are linearly independent over $\mathbf{Q}$. Then for any $\delta>0$, there exists $g \in G L_{n}(\mathbf{Z})$ such that $\|\boldsymbol{y} g\|<\delta$.

Proof. We write $\ell(\boldsymbol{x})=\min _{1 \leqslant i \leqslant n}\left|x_{i}\right|$ for $\boldsymbol{x}=\left(x_{1}, \ldots, x_{n}\right) \in \mathbf{R}^{n}$. Note that for all $g \in G L_{n}(\mathbf{Z})$, no entry of $\boldsymbol{y} g$ is zero because of the linear independence over $\mathbf{Q}$. In particular $\ell(\boldsymbol{y} g)>0$ for all $g \in G L_{n}(\mathbf{Z})$.

For a $\boldsymbol{z}=\boldsymbol{y} g$, we take an index $i_{0}$ such that $\ell(\boldsymbol{z})=\left|z_{i_{0}}\right|$. For each $j \neq i_{0}$, there are a $q_{j} \in \mathbf{Z}$ and a $z_{j}^{\prime} \in \mathbf{R}$ such that $z_{j}=q_{j} z_{i_{0}}+z_{j}^{\prime}$ with 
$\left|z_{j}^{\prime}\right| \leqslant\left|z_{i_{0}}\right| / 2$. We take $q_{i_{0}}=0$ and $z_{i_{0}}^{\prime}=z_{i_{0}}$. We set $\boldsymbol{z}^{\prime}=\left(z_{1}^{\prime}, \ldots, z_{n}^{\prime}\right) \in \mathbf{R}^{n}$ and

$$
g^{\prime}=\prod_{j=1}^{n}\left(I+E_{i_{0}, j}\right)^{q_{j}}=I+\sum_{j \neq i_{0}} q_{j} E_{i_{0}, j} \in G L_{n}(\mathbf{Z}),
$$

where $E_{i j}$ is the matrix unit. Then this $\boldsymbol{z}^{\prime}$ satisfies that $\boldsymbol{z}^{\prime} g^{\prime}=\boldsymbol{z},\left\|\boldsymbol{z}^{\prime}\right\|=$ $\left|z_{i_{0}}\right|=\ell(\boldsymbol{z})$ and $\ell\left(\boldsymbol{z}^{\prime}\right) \leqslant\left|z_{i_{0}}\right| / 2=\ell(\boldsymbol{z}) / 2$.

Repeating this process, we obtain an element $\boldsymbol{z}$ in the $G L_{n}(\mathbf{Z})$-orbit of $\boldsymbol{y}$ such that $\ell(\boldsymbol{z})<\delta$. Finally, again by the construction above, we have $\left\|\boldsymbol{z}^{\prime}\right\|=\ell(\boldsymbol{z})<\delta$. This $\boldsymbol{z}^{\prime}$ is the desired element contained in the $G L_{n}(\mathbf{Z})$ oribit.

We will apply Proposition 8 to our situation later.

\subsection{The group $\Gamma$}

We set

$$
\Gamma(2)=\Gamma_{n}(2)=\left\{\left(a_{i j}\right) \in G L_{n}(\mathbf{Z}) \mid a_{i j} \equiv \delta_{i j} \bmod 2 \text { for all } i, j\right\} .
$$

Let $\Delta_{n}$ be the set of diagonal matrices in $G L_{n}(\mathbf{Z})$, that is,

$$
\Delta_{n}=\left\{\operatorname{diag}\left(\varepsilon_{1}, \ldots, \varepsilon_{n}\right) \mid \varepsilon_{1}, \ldots, \varepsilon_{n} \in\{1,-1\}\right\} .
$$

We define $\Gamma=\Gamma_{n}$ to be the subgroup of $G L_{n}(\mathbf{Z})$ generated by all $I+2 E_{i j}$ with $i \neq j$. Then $\Delta_{n} \subset \Gamma_{n}(2)$ and $\Gamma_{n} \subset \Gamma_{n}(2)$.

We set

$$
X:=\left\{\begin{array}{l|l}
\boldsymbol{x}=\left(x_{1}, \ldots, x_{n}\right) \in \mathbf{Z}^{n} & \begin{array}{l}
x_{1}-1, x_{2}, \ldots, x_{n} \in 2 \mathbf{Z} \\
\boldsymbol{x} \notin m \mathbf{Z}^{n} \text { for all } m \in \mathbf{Z}_{>1}
\end{array}
\end{array}\right\} .
$$

It is easy to see that the group $\Gamma(2)$ acts on $X$. We give the orbit decomposition of the action of $\Gamma \subset \Gamma(2)$ on $X$.

Lemma 10. We have $X=\mathbf{e}_{1} \Gamma \cup\left(-\mathbf{e}_{1}\right) \Gamma$, where $\mathbf{e}_{1}=(1,0, \ldots, 0) \in X$.

Proof. For $\boldsymbol{x} \in X$, there exists an index $i_{0}$ such that $x_{i_{0}} \neq 0$ and $\left|x_{j}\right| \geqslant\left|x_{i_{0}}\right|$ unless $x_{j}=0$. For each $j \neq i_{0}$, there exist integers $q_{j}$ and $x_{j}^{\prime}$ such that $x_{j}=2 q_{j} x_{i_{0}}+x_{j}^{\prime}$ with $\left|x_{j}^{\prime}\right| \leqslant\left|x_{i_{0}}\right|$. We take $q_{i_{0}}=0$ and $x_{i_{0}}^{\prime}=x_{i_{0}}$. Also we take $q_{j}=x_{j}^{\prime}=0$ if $x_{j}=0$. This implies that $\left|x_{j}^{\prime}\right| \leqslant\left|x_{j}\right|$ for all $j$. We set $\boldsymbol{x}^{\prime}=\left(x_{1}^{\prime}, \ldots, x_{n}^{\prime}\right)$ and $\gamma=I+\sum_{j=1}^{n} 2 q_{j} E_{i_{0}, j}=\prod_{j=1}^{n}\left(I+2 E_{i_{0}, j}\right)^{q_{j}}$. 
Then we have $\boldsymbol{x}^{\prime} \in X, \gamma \in \Gamma$, and $\boldsymbol{x}^{\prime} \gamma=\boldsymbol{x}$. That is, $\boldsymbol{x}$ and $\boldsymbol{x}^{\prime}$ belong to the same $\Gamma$-orbit. We see that

$$
\|\boldsymbol{x}\|=\max _{1 \leqslant i \leqslant n}\left|x_{i}\right| \geqslant \min _{1 \leqslant i \leqslant n}\left(\left|x_{i}\right| \neq 0\right)=\left|x_{i_{0}}\right|=\max _{1 \leqslant i \leqslant n}\left|x_{i}^{\prime}\right|=\left\|\boldsymbol{x}^{\prime}\right\| .
$$

The equality $\|\boldsymbol{x}\|=\left\|\boldsymbol{x}^{\prime}\right\|$ holds only for $\boldsymbol{x}= \pm \mathbf{e}_{1}$. In fact, in such a case, $x_{2}=\cdots=x_{n}=0$ since $x_{1}$ is odd and others are even. This implies that $\boldsymbol{x}$ is a multiple of $\mathbf{e}_{1}$. By the condition $\boldsymbol{x} \notin m \mathbf{Z}^{n}$ for $m>1$, we have $\boldsymbol{x}= \pm \mathbf{e}_{1}$.

Note that $e_{1} \Gamma$ and $\left(-e_{1}\right) \Gamma$ are distinct since $\left( \pm e_{1}\right) \Gamma \subset\left\{x \in X \mid x_{1} \in\right.$ $\pm 1+4 \mathbf{Z}\}$.

Proposition 11. For $g \in \Gamma_{n}(2)$, there is a $\gamma \in \Gamma_{n}$ such that $g \gamma \in \Delta_{n}$.

Proof. Take a $g \in \Gamma_{n}(2)$. We will construct such a $\gamma \in \Gamma_{n}$ by induction on the size " $n$ ". Set $\boldsymbol{x}=\mathbf{e}_{1} g$ then we see that $\boldsymbol{x} \in X$. By Lemma 10, there exists $\gamma_{1} \in \Gamma_{n}$ and $\varepsilon_{1}= \pm 1$ such that $\boldsymbol{x} \gamma_{1}=\varepsilon_{1} \mathbf{e}_{1}$. Then $g \gamma_{1}=\left[\begin{array}{c|c}\varepsilon_{1} & 0 \\ \hline * & g_{2}\end{array}\right]$ where $g_{2} \in M_{n-1}$. The form above and $g \gamma_{1} \in \Gamma_{n}(2)$ imply that $g_{2} \in \Gamma_{n-1}(2)$. By induction hypothesis we have $\gamma_{2}^{\prime} \in \Gamma_{n-1}$ such that $t_{2}:=g_{2} \gamma_{2}^{\prime} \in \Delta_{n-1}$. We set $\gamma_{2}=\left[\begin{array}{c|c}1 & 0 \\ \hline 0 & \gamma_{2}^{\prime}\end{array}\right] \in \Gamma_{n}, t=\left[\begin{array}{c|c}\varepsilon_{1} & 0 \\ \hline 0 & t_{2}\end{array}\right] \in \Delta_{n}$ and $\gamma_{3}:=t^{-1} g \gamma_{1} \gamma_{2}$. Then $\gamma_{3}$ is of the form $\left[\begin{array}{c|c}1 & 0 \\ \hline{ }^{t} v & I_{n-1}\end{array}\right]$ and the property $\gamma_{3} \in \Gamma_{n}(2)$ implies that $v=\left(2 q_{2}, \ldots, 2 q_{n}\right) \in 2 \mathbf{Z}^{n-1}$. Thus $\gamma_{3}=\prod_{i=2}^{n}\left(I+2 E_{i, 1}\right)^{q_{i}} \in \Gamma_{n}$ and we finally see that $\gamma:=\gamma_{1} \gamma_{2} \gamma_{3}^{-1} \in \Gamma_{n}$. Hence we have the desired assertion $g \gamma=t$.

COROLlary 12. (i) The map $\Delta_{n} \times \Gamma \ni(t, \gamma) \mapsto t \gamma \in \Gamma(2)$ is surjective.

(ii) The group $\Gamma \subset G L_{n}(\mathbf{Z})$ is of finite index.

(iii) Any $\Gamma$-invariant continuous function on $\mathbf{R}^{n}$ is constant.

Proof. (i) is a paraphrase of the proposition above. (ii) follows from (i) and the fact that $\Gamma(2)$ is of index finite in $G L_{n}(\mathbf{Z})$. (iii) follows from (ii) and Proposition 8. 


\subsection{The group generated by $W$ and $\bar{W}$}

We denote by $\langle W, \bar{W}\rangle$ the subgroup of $G L_{3}(\mathbf{Q})$ generated by $W$ and $\bar{W}=Q^{-1} W Q$.

Lemma 13. $\Gamma_{3} \subset\langle W, \bar{W}\rangle$.

Proof. We will construct generators of $\Gamma_{3}$ explicitly. Recall that $T=$ $(13) \in \mathfrak{S}_{3}$ and $C=(123) \in \mathfrak{S}_{3}$ are regarded as elements in $G L_{3}(\mathbf{Q})$. We set

$$
g_{1}:=\left(Q^{-1} C T Q\right) C \in \bar{W} W \subset\langle W, \bar{W}\rangle .
$$

By an explicit computation, we know that $N_{1}:=g_{1}^{2}-I \in M_{3}(\mathbf{Q})$ satisfies $N_{1}^{2}=O$. We set $N_{2}=\operatorname{diag}(-1,1,1) N_{1} \operatorname{diag}(-1,1,1)$, then $N_{2}^{2}=N_{1} N_{2}=$ $N_{2} N_{1}=O$. We set $N_{3}=N_{1}-N_{2}$, and $N_{4}=\operatorname{diag}(1,1,-1) N_{3} \operatorname{diag}(1,1,-1)$, then $N_{3}^{2}=N_{4}^{2}=N_{3} N_{4}=N_{4} N_{3}=O$. We set $N_{5}=N_{4}-N_{3}$. These relations show that

$$
\begin{aligned}
I+N_{1}, I+N_{2}, I+N_{3}=\left(I+N_{1}\right)\left(I+N_{2}\right)^{-1} \\
I+N_{4}, I+N_{5}=\left(I+N_{4}\right)\left(I+N_{3}\right)^{-1} \in\langle W, \bar{W}\rangle .
\end{aligned}
$$

To be more explicit,

$$
\begin{aligned}
g_{1} & =\left[\begin{array}{ccc}
-1 / 2 & 1 / 2 & 1 / 2 \\
1 & 1 & 0 \\
1 / 2 & -1 / 2 & 1 / 2
\end{array}\right] \\
N_{1} & =\left[\begin{array}{ccc}
0 & 0 & 0 \\
1 / 2 & 1 / 2 & 1 / 2 \\
-1 / 2 & -1 / 2 & -1 / 2
\end{array}\right]=\frac{1}{2}\left[\begin{array}{c}
0 \\
1 \\
-1
\end{array}\right]\left[\begin{array}{lll}
1 & 1 & 1
\end{array}\right],
\end{aligned}
$$

$N_{3}=E_{21}-E_{31}, N_{4}=E_{21}+E_{31}$, and $N_{5}=2 E_{31}$. Hence we have $I+2 E_{31} \in$ $\langle W, \bar{W}\rangle$.

Finally, using the conjugation by permutation matrices, we have $I+$ $2 E_{i j} \in\langle W, \bar{W}\rangle$ for all $i \neq j$. This proves $\Gamma_{3} \subset\langle W, \bar{W}\rangle$.

Corollary 14. (i) Any $\langle W, \bar{W}\rangle$-invariant continuous function on $\mathbf{R}^{3}$ is constant.

(ii) $\mathbf{Q}[\boldsymbol{x}]^{W} \cap \mathbf{Q}[\boldsymbol{x}]^{\bar{W}}=\mathbf{Q}$.

Proof. (i) follows from (iii) of Corollary 12 and Lemma 13. (ii) follows from (i) and the definition of $\langle W, \bar{W}\rangle$. 
Remark 15. There is another way to derive the statement (ii) from the fact that $\Gamma_{n}$ is Zariski dense in $S L_{n}(\mathbf{C})$. In fact, $\mathbf{C}[\boldsymbol{x}]^{\langle W, \bar{W}\rangle}=\mathbf{C}[\boldsymbol{x}]^{\overline{\langle W, \bar{W}\rangle}} \subset$ $\mathbf{C}[\boldsymbol{x}]^{\overline{\Gamma_{n}}}=\mathbf{C}[\boldsymbol{x}]^{S L_{n}(\mathbf{C})}=\mathbf{C}$, where $\overline{\langle W, \bar{W}\rangle}$ and $\overline{\Gamma_{n}}$ stand for the Zariski closure of $\langle W, \bar{W}\rangle$ and $\Gamma_{n}$ respectively. Any $\langle W, \bar{W}\rangle$-invariant polynomial is also invariant under the Zariski closure of $\langle W, \bar{W}\rangle$, which contains $S L_{n}(\mathbf{C})$ by Lemma 13. Then such a polynomial is constant since $S L_{n}(\mathbf{C})$ has an open dense orbit $\mathbf{C}^{n} \backslash\{0\}$ on $\mathbf{C}^{n}$.

\section{$\S 4$. The dimension of $D S h_{3}(d)$}

Recall that $\mathcal{Z}=\bigoplus_{k \geqslant 0} \mathcal{Z}_{k}$ is the graded algebra generated by all MZV's over $\mathbf{Q}$, where $\mathcal{Z}_{k}$ is the $\mathbf{Q}$-vector space generated by MZV's of weight $k$. The algebra $\mathcal{Z}$ has a filtration defined by $\mathcal{Z}=\bigcup_{n \geqslant 0} \mathcal{Z}^{(n)}$ where $\mathcal{Z}^{(n)}=$ $\bigoplus_{k \geqslant 0} \mathcal{Z}_{k}^{(n)}$ and $\mathcal{Z}_{k}^{(n)}$ is the $\mathbf{Q}$-vector space spanned by MZV's of weight $k$ and depth $\leqslant n$.

Let $D_{k, n}$ be the number of algebra generators of $\mathcal{Z}$ which sit in the part of weight $k$ and depth $n$. As mentioned in Introduction, there is a conjectural formula due to Broadhurst and Kreimer, which gives $D_{k, n}$ :

Conjecture 16. ([1]) Define the numbers $\bar{D}_{k, n}$ by $\bar{D}_{2,1}:=1$ and by the equation:

$$
\prod_{\substack{k \geqslant 3 \\ n \geqslant 1}}\left(1-x^{k} y^{n}\right)^{\bar{D}_{k, n}}=1-\frac{x^{3} y}{1-x^{2}}+\frac{x^{12} y^{2}\left(1-y^{2}\right)}{\left(1-x^{4}\right)\left(1-x^{6}\right)}
$$

then $\bar{D}_{k, n}=D_{k, n}$ hold for all $k \geqslant 2, n \geqslant 1$.

Remark 17. In general, it is out of reach at present to show the inequality $\bar{D}_{k, n} \leqslant D_{k, n}$. For example, $D_{5,1} \neq 0$ (note that $\bar{D}_{5,1}=1$ ) implies the algebraic independence among $\zeta(2), \zeta(3)$ and $\zeta(5)$ over $\mathbf{Q}$, but nobody has been able to prove this.

Picking up the coefficients of $y^{3}$ in (6) (taking the logarithmic differential for $y$ and use the Möbius inversion formula), we have a generating function

$$
\sum_{d>0} \bar{D}_{d+3,3} x^{d}=\frac{x^{8}\left(1+x^{2}-x^{4}\right)}{\left(1-x^{2}\right)\left(1-x^{4}\right)\left(1-x^{6}\right)}=\sum_{d>0, d \in 2 \mathbf{Z}}\left[\frac{d^{2}-1}{48}\right] x^{d} .
$$


As mentioned in Introduction, Goncharov shows the inequality $D_{d+3,3} \leqslant$ $\bar{D}_{d+3,3}$ in [2]. In the following, we can give the same estimate, by quite different method, using the theory of invariants for reflection groups.

For $d>0$, let us define the map $\varpi_{(d)}: \mathbf{Q}[\boldsymbol{x}]_{(d)}^{W^{\prime}} \rightarrow \mathbf{Q}[\boldsymbol{x}]_{(d)}^{W} \oplus \mathbf{Q}[\boldsymbol{x}]_{(d)}^{\bar{W}}$ by $\varpi_{(d)}(g)=\left(\pi_{(d)}(g), \bar{\pi}_{(d)}(g)\right)=\left(g\left|s h_{1}, g\right| Q^{-1} s h_{1} Q\right)$. Since ker $\varpi_{(d)}=$ $\operatorname{ker} \pi_{(d)} \cap \operatorname{ker} \bar{\pi}_{(d)}$, we have the isomorphism $D S h_{3}(d) \simeq \operatorname{ker} \varpi_{(d)}(f \mapsto g=$ $f \mid R)$ for even $d$ by Theorem 7 . So we obtain an exact sequence:

$$
0 \longrightarrow D S h_{3}(d) \stackrel{R}{\longrightarrow} \mathbf{Q}[\boldsymbol{x}]_{(d)}^{W^{\prime}} \stackrel{\varpi_{(d)}}{\longrightarrow} \mathbf{Q}[\boldsymbol{x}]_{(d)}^{W} \oplus \mathbf{Q}[\boldsymbol{x}]_{(d)}^{\bar{W}} .
$$

THEOREM 18. For even $d>0$, the following is an exact sequence:

$$
0 \longrightarrow D S h_{3}(d) \stackrel{R}{\longrightarrow} \mathbf{Q}[\boldsymbol{x}]_{(d)}^{W^{\prime}} \stackrel{\varpi_{(d)}}{\longrightarrow} \mathbf{Q}[\boldsymbol{x}]_{(d)}^{W} \oplus \mathbf{Q}[\boldsymbol{x}]_{(d)}^{\bar{W}} \longrightarrow 0 .
$$

Proof. It is enough to show that $\varpi_{(d)}$ is surjective, which is equivalent to show the injectivity of the dual map of $\varpi_{(d)}$ :

$$
\left(\varpi_{(d)}\right)^{*}:\left(\mathbf{Q}[\boldsymbol{x}]_{(d)}^{W}\right)^{*} \oplus\left(\mathbf{Q}[\boldsymbol{x}]_{(d)}^{\bar{W}}\right)^{*} \longrightarrow\left(\mathbf{Q}[\boldsymbol{x}]_{(d)}^{W^{\prime}}\right)^{*} .
$$

If we identify the dual of invariant space for a finite group with the invariants of dual space: $\left(\mathbf{Q}[\boldsymbol{x}]_{(d)}^{*}\right)^{W} \cong\left(\mathbf{Q}[\boldsymbol{x}]_{(d)}^{W}\right)^{*}$ via the composition map $\left(\mathbf{Q}[\boldsymbol{x}]_{(d)}^{*}\right)^{W} \rightarrow \mathbf{Q}[\boldsymbol{x}]_{(d)}^{*} \rightarrow\left(\mathbf{Q}[\boldsymbol{x}]_{(d)}^{W}\right)^{*}$, where the first map is the natural inclusion and the second is the dual map of the inclusion $\mathbf{Q}[\boldsymbol{x}]_{(d)}^{W} \subset \mathbf{Q}[\boldsymbol{x}]_{(d)}$ (resp. for $\bar{W}$ and $W^{\prime}$ ), then it is easy to check that the map $\left(\varpi_{(d)}\right)^{*}$ : $\left(\mathbf{Q}[\boldsymbol{x}]_{(d)}^{*}\right)^{W} \oplus\left(\mathbf{Q}[\boldsymbol{x}]_{(d)}^{*}\right)^{\bar{W}} \rightarrow\left(\mathbf{Q}[\boldsymbol{x}]_{(d)}^{*}\right)^{W^{\prime}}$ is induced from the inclusion maps $\left(\mathbf{Q}[\boldsymbol{x}]_{(d)}^{*}\right)^{W} \subset\left(\mathbf{Q}[\boldsymbol{x}]_{(d)}^{*}\right)^{W^{\prime}}$ and $\left(\mathbf{Q}[\boldsymbol{x}]_{(d)}^{*}\right)^{\bar{W}} \subset\left(\mathbf{Q}[\boldsymbol{x}]_{(d)}^{*}\right)^{W^{\prime}}$. So the triviality of the intersection of the spaces $\left(\mathbf{Q}[\boldsymbol{x}]_{(d)}^{*}\right)^{W}$ and $\left(\mathbf{Q}[\boldsymbol{x}]_{(d)}^{*}\right)^{\bar{W}}$ in $\mathbf{Q}[\boldsymbol{x}]_{(d)}^{*}$ implies the injectivity of $\left(\varpi_{(d)}\right)^{*}$. It can be proved by Corollary 14 (ii), since $\left(\mathbf{Q}[\boldsymbol{x}]_{(d)}^{*}\right)^{\langle W, \bar{W}\rangle}$ is isomorphic to $\mathbf{Q}[\boldsymbol{x}]_{(d)}^{\langle W, \bar{W}\rangle}$.

Theorem 19. For even $d>0$, we have $\operatorname{dim} D S h_{3}(d)=\bar{D}_{d+3,3}=$ $\left[\frac{d^{2}-1}{48}\right]$ and consequently $D_{d+3,3} \leqslant \bar{D}_{d+3,3}$.

Proof. From (2) and (3), we have generating functions

(9) $\sum_{d \geqslant 0} \operatorname{dim} \mathbf{Q}[\boldsymbol{x}]_{(d)}^{W} x^{d}=\sum_{d \geqslant 0} \operatorname{dim} \mathbf{Q}[\boldsymbol{x}]_{(d)}^{\bar{W}} x^{d}=\frac{1}{\left(1-x^{2}\right)\left(1-x^{4}\right)\left(1-x^{6}\right)}$,

$$
\sum_{d \geqslant 0} \operatorname{dim} \mathbf{Q}[\boldsymbol{x}]_{(d)}^{W^{\prime}} x^{d}=\frac{1}{\left(1-x^{2}\right)^{2}\left(1-x^{4}\right)} .
$$


By (8), we have

$$
\operatorname{dim} D S h_{3}(d)=\operatorname{dim} \mathbf{Q}[\boldsymbol{x}]_{(d)}^{W^{\prime}}-2 \operatorname{dim} \mathbf{Q}[\boldsymbol{x}]_{(d)}^{W}
$$

for $d>0$. Hence we obtain the generating function of $\operatorname{dim} D S h_{3}(d)$ :

$$
\begin{aligned}
& \sum_{d>0} \operatorname{dim} D S h_{3}(d) x^{d} \\
& \quad=\left(\frac{1}{\left(1-x^{2}\right)^{2}\left(1-x^{4}\right)}-1\right)-2\left(\frac{1}{\left(1-x^{2}\right)\left(1-x^{4}\right)\left(1-x^{6}\right)}-1\right) \\
& \quad=\frac{x^{8}\left(1+x^{2}-x^{4}\right)}{\left(1-x^{2}\right)\left(1-x^{4}\right)\left(1-x^{6}\right)},
\end{aligned}
$$

which coincides with (7). This proves the first claim. Apply this to the result $D_{d+n, n} \leqslant \operatorname{dim} D S h_{n}(d)$ in [8], we have the last claim in the theorem. This completes the proof.

Acknowledgement. The authors would like to thank to Professor Seidai Yasuda in RIMS. He suggested a method of the proof of Theorem 18 and gave valuable advices to them.

\section{REFERENCES}

[1] D. J. Broadhurst and D. Kreimer, Association of multiple zeta values with positive knots via Feynman diagrams up to 9 loops, Physics Lett. B, 393 (1997), 403-412.

[2] A. B. Goncharov, Multiple polylogarithms, cyclotomy and modular complexes, Math. Res. Lett., 5 (1998), 497-516.

[3] A. B. Goncharov, Multiple $\zeta$-values, Galois groups and geometry of modular varieties, Progr. Math. 201, Birkhäuser, 2001, pp. 361-392.

[4] A. B. Goncharov, The dihedral Lie algebras and Galois symmetries of $\pi_{1}^{(l)}\left(\mathbb{P}^{1}-\right.$ $\left.\left(\{0, \infty\} \cup \mu_{N}\right)\right)$, Duke Math. J., 110 (2001), 397-487.

[5] A. B. Goncharov, Multiple polylogarithms and mixed Tate motives, preprint (2001), math. AG/0103059.

[6] J. E. Humphreys, Reflection groups and Coxeter Groups, Cambridge, 1990.

[7] K. Ihara, Derivations and double shuffle relations for multiple zeta values, RIMS Kokyuroku, 1549 (2007), 47-63.

[8] K. Ihara, M. Kaneko, D. Zagier, Derivations and double shuffle relations for multiple zeta values, Compositio Math., 142 (2006), 307-338.

[9] D. Zagier, Periods of modular forms, trace of Hecke operators, and multiple zeta values, RIMS Kokyuroku, 843 (1993), 162-170.

[10] D. Zagier, Values of zeta functions and their applications, Progr. Math. 120, Birkhäuser, 1994, pp. 497-512. 
Kentaro Ihara

Department of Mathematics Graduate School of Science

Osaka University

Machikaneyama

Toyonaka Osaka 560-0043

Japan

k-ihara@math.sci.osaka-u.ac.jp

Hiroyuki Ochiai

Department of Mathematics

Nagoya University

Chikusa

Nagoya 464-8602

Japan

ochiai@math.nagoya-u.ac.jp 